\title{
Eficiência técnica e econômica do controle biológico da traça-do-toma- teiro em ambiente protegido
}

\author{
Maria Alice de Medeiros ${ }^{1}$; Nirlene J Vilela ${ }^{1}$; Félix Humberto França ${ }^{2}$ \\ ${ }^{1}$ Embrapa Hortaliças, C. Postal 218, 70359-970 Brasília-DF; ${ }^{2}$ Embrapa Sede/SPD, Brasília-DF; E-mail: medeiros@ @nph.embrapa.br; \\ nirlene@cnph.embrapa.br; felix.franca@sede.embrapa.br
}

\section{RESUMO}

A eficiência técnica e econômica do controle da traça-do-tomateiro (Tuta absoluta) pelo parasitóide Trichogramma pretiosum foi avaliada em ambiente protegido na área de um produtor do município de Luziânia-GO, durante o verão de 1999-2000. O trabalho foi conduzido em duas casas de vegetação, sendo cada uma delas submetida aos seguintes regimes de controle de pragas: (1) prática do produtor: consistiu em pulverizações de inseticidas de acordo com a necessidade, em rotação com os produtos deltametrina, abamectin, Bacillus thuringiensis (nas doses recomendadas) e detergente $0,5 \%$; (2) liberações inundativas de T. pretiosum associada com pulverizações semanais de $B$. thuringiensis em formulação concentrada emulsionável (na dose recomendada). As medidas de controle foram iniciadas logo após a constatação dos primeiros adultos da traça-do-tomateiro. A liberação massal consistiu na colocação de duas cartelas por semana $(20 \times 30 \mathrm{~cm})$ de ovos parasitados por T. pretiosum que foram gradualmente aumentadas até que atingisse seis cartelas por semana por ocasião da colheita do tomate. Semanalmente, foram coletados 50 folíolos de tomateiro ao acaso em cada casa de vegetação. O número de ovos foi determinado, em laboratório e mantidos em câmaras climatizadas para a constatação das lagartas ou parasitismo. A produtividade e o dano nos frutos foram determinados semanalmente em 50 tomateiros escolhidos ao acaso em cada tratamento. A produtividade com controle biológico foi de 6.160 bandejas/600g e com controle químico foi de 6.833 bandejas/600g. Os custos variáveis da produção foram de R \$ 3,9 mil e R \$ 4,7 mil para o sistema com controle biológico e químico, respectivamente. O limite de quebra de safra permitido para ambos sistemas de produção, indicado pela margem de segurança econômica, foi maior para o controle biológico $(61,7 \%)$. O coeficiente de eficiência econômica do controle biológico foi significativo $(2,62)$ e a taxa interna de retorno $(24,9 \%)$ confirmou a viabilidade econômica do sistema de produção de tomate utilizando o controle biológico com $T$. pretiosum.

Palavras-chave: Tuta absoluta, Lycopersicon esculentum, Trichogramma pretiosum, Bacillus thuringiensis.

\begin{abstract}
Technical and economic efficiency of biological control of the South American tomato pinworm in protected environment

The economic and technical efficiency of controlling the South American tomato pinworm (Tuta absoluta) by the parasitoid Trichogramma pretiosum was evaluated in protected environment, in Luziania, Goiás State, Brazil, during the summer of 1999-2000. The research was conducted in two greenhouses, each one submitted to the following pest control regimes: (1) habitual farmer practices: pesticides sprayed based on presence/absence of the pest and rotation of products (deltamethrin; abamectin; Bacillus thuringiensis (at the recommended dose); and soap at $0,5 \%$ concentration). (2) inundative release of $T$. pretiosum associated with weekly sprays of Bacillus thuringiensis as an emulsifiable concentrate formulation (at the recommended dose). Insecticide sprays and parasitoid mass release were initiated just after the first adults were observed. Mass release consisted of weekly display of two cards $(20 \times 30 \mathrm{~cm})$ of parasitized eggs by $T$. pretiosum that were gradually increased until the reached six cards each week, by the time of tomato harvest. Once a week, 50 leaflets were collected in each greenhouse. The number of eggs was determined in the laboratory and kept inside chambers to determine the number of larvae or the percentage of parasitism. Tomato production and damage to the fruits were determined weekly from 50 tomato plants randomly chosen in each treatment. The productivity of the treatment using biological control was 6,160 polystyrene trays $/ 600 \mathrm{~g}$ and 6,833 trays $/ 600 \mathrm{~g}$ when chemical control was used. The variable costs of production were $\mathrm{R} \$ 3,9$ thousands and $\mathrm{R} \$ 4,7$ thousands for the biological control and chemical control, respectively. The loss limit in each cropping season for both production systems, estimated by the technical-economic efficiency, was higher for the biological control $(61,7 \%)$. The coefficient of economic efficiency was significant (2.62) and the internal rate of return $(24.9 \%)$ confirmed that the system of production that used the biological control by $T$. pretiosum was economically efficient.
\end{abstract}

Keywords: Tuta absoluta, Lycopersicon esculentum, Trichogramma pretiosum, Bacillus thuringiensis.

(Recebido para publicação em 24 de maio de 2005; aceito em 31 de maio de 2006)

$\mathrm{N}$ o Brasil, o tomate é uma cultura de elevada importância socioeconômica. Além de ser um alimento substancialmente nutritivo, é uma fonte de geração de emprego e renda na agricultura. No grupo das hortaliças, o tomate de mesa é a cultura mais exigente em mão-de-obra, tanto nos sistemas de produção em campo aberto quanto em sis- temas protegidos, estimando uma geração de 4 a 5 empregos diretos por hectare/ano.

A traça-do-tomateiro, Tuta absoluta (Meyrick) (Lepidoptera: Gelechiidae), é a principal praga do tomate no Distrito Federal e região geo-econômica. Desde que foi introduzida no Brasil, no final dos anos setenta, tem causado gran- des prejuízos danificando folhas, hastes e frutos do tomateiro (França et al., 2000). Visando o controle da praga, agrotóxicos são pulverizados até vinte vezes durante o ciclo da cultura, o que representa de $12 \%$ a $25 \%$ dos custos de produção do tomate. Apesar das pulverizações intensivas, $30 \%$ dos frutos são impróprios para o consumo (França, 
1993). Além dos riscos para a saúde humana, os inseticidas químicos também causam fortes desequilíbrios ao meio ambiente, destruindo os inimigos naturais das diferentes pragas da cultura nas áreas sob controle.

Uma alternativa aos inseticidas é o controle biológico, por meio da utilização do parasitóide Trichogramma pretiosum Ridley (Hymenoptera: Trichogrammatidae) associado com aplicações do entomopatógeno Bacillus thuringiensis Berliner (Haji et al., 1995). Esta técnica pode reduzir em até $70 \%$ o custo de controle da praga, aumentando a produção de frutos sadios e reduzindo a contaminação ambiental (França, 1993).

A viabilidade técnica do controle biológico para a traça-do-tomateiro já foi confirmada em outros trabalhos (Haji et al., 1995). No entanto, para que o controle biológico seja adotado pelos produtores são necessários estudos sobre as vantagens técnico-econômicas do sistema. Diversos métodos vem sendo usados na avaliação de negócios agrícolas, como por exemplo, o método de orçamentação parcial tem sido utilizado para realização de análises estáticas comparativas de sistemas de produção (Perrin et al., 1985; Scolari et. al. 1985; Snodgrass \& Wallace, 1993) e modelos intertemporais para análise de viabilidade econômica (Hirschfeld, 1998; Pearce, 1983; Laiard \& Glaister, 1996; Laponni, 1996; Andrews \& Reganold, 2000; Antle \& Stoorvogel, 2005). A taxa interna de retorno (TIR) é o indicador mais comum em estudos de viabilidade econômica, e pode ser usada para mostrar o retorno dos recursos alocados nos negócios agrícolas. A taxa interna de retorno superior ao custo de oportunidade do capital investido no mercado financeiro, ou de capitais indica a viabilidade econômica do negócio. O tempo de recuperação dos investimentos nos dois sistemas de produção também é importante e pode ser determinado pelo método do 'pay-back simples' (PBS) (Hirschfeld, 1998). O índice de lucratividade (IL) representa um critério para seleção de alternativas econômicas (Laponni, 1996).

O objetivo deste trabalho foi avaliar a eficiência técnica-econômica do controle da traça-do-tomateiro com o parasitóide T. pretiosum, associado com aplicações do entomopatógeno $B$. thuringiensis em plantas de tomate cultivadas em ambiente protegido.

\section{MATERIAL E MÉTODOS}

Uma unidade de observação da tecnologia de controle biológico da traça-do-tomateiro utilizando T. pretiosum foi instalada na área de um produtor de tomate, no município de Luziânia-GO. $\mathrm{O}$ trabalho foi conduzido durante o verão de 1999-2000 em duas casas de vegetação. O produtor encarregou-se das tarefas de criar e liberar o T. pretiosum de acordo com as recomendações da Embrapa Hortaliças. As casas de vegetação eram do tipo não climatizadas, de $10 \times 50 \mathrm{~m}$, e cobertas com tela clarite (intercepção de 80\%). A cultivar de tomate para mesa utilizada foi 'Grande Uhur', com densidade de 1980 plantas em espaçamentos de $30 \times 40 \mathrm{~cm}$. Os seguintes regimes de controle de insetos foram estabelecidos em cada casa de vegetação: (1) prática usual do produtor, que consistiu em pulverizações de acordo com a necessidade, em rotação dos seguintes produtos: $B$. thuringiensis, abamectin + óleo mineral, deltametrina (nas doses recomendadas) e detergente $0,5 \%$ (indicado para mosca-branca) (Tabela 1); (2) liberações inundativas de $T$. pretiosum (criado em Sitotroga cerealella (Olivier) como hospedeiro) (Haji et. al., 1998) associada com pulverizações semanais de $B$. thuringiensis em formulação concentrada emulsionável, na dose recomendada. As pulverizações com inseticidas e as liberações massais de parasitóides foram iniciadas logo após a detecção dos primeiros adultos da traça-do-tomateiro. A liberação massal do parasitóide empregou inicialmente duas cartelas por semana $(20 \times 30 \mathrm{~cm})$ com ovos parasitados por $T$. pretiosum, próximo à emergência dos adultos, que foram gradualmente aumentadas até atingir seis cartelas por semana, por ocasião da colheita do tomate. As cartelas foram distribuídas em pedaços de $6,45 \mathrm{~cm}$ ou $1 \mathrm{pol}^{2}$ e dispostas entre os fitilhos do tutoramento. Semanalmente, foram coletados 50 folíolos de tomateiros para determinar o número de ovos da traça-do-tomatei- ro. Em laboratório, os ovos coletados foram individualizados em cápsulas de gelatina, mantidos em câmaras climatizadas tipo BOD a $25 \pm 2^{\circ} \mathrm{C}, 70 \%$ UR e $14 \mathrm{~h}$ de fotofase para determinar o índice de parasitismo nos ovos da traça-do-tomateiro. A produtividade e a percentagem de dano causado foi estimada pelo número de frutos produzidos em 50 plantas escolhidas ao acaso nos dois tratamentos, desde o início até o final da colheita, considerando uma colheita por semana. Além dos métodos de controle descritos, o produtor adotou as seguintes práticas: as casas de vegetação foram protegidas com tela anti-afídeos, com compartimento de isolamento na entrada; foram utilizados substrato novo e mudas sadias no transplante, não infestadas com traça-do-tomateiro; o controle químico foi empregado de forma racional, ou seja, de acordo com a necessidade, considerando as amostragens semanais. Os trabalhos foram acompanhados semanalmente por pesquisadores da Embrapa e visitados por outros produtores da região.

Os custos de produção foram determinados com base nos coeficientes técnicos para cada sistema de produção multiplicados pelos preços de mercado dos fatores produtivos. A receita foi determinada pela quantidade vendida aos preços pagos ao produtor (Tabela 1). A análise econômica foi processada pelo programa de análise pelo método de orçamentação parcial (MOP). Adicionalmente, empregou-se um modelo intertemporal para análise de viabilidade econômica e dos impactos potenciais ex-ante gerados pelo sistema de controle biológico, considerando o horizonte de 5 anos. Além dos indicadores econômicos básicos (receita, custos variáveis, custos fixos, margens do produtor, eficiência, ponto de equilíbrio da produção, margens de segurança) fornecidos pelas análises por orçamentação parcial foi calculada a taxa interna de retorno (TIR).

A TIR foi calculada por:

$$
\sum_{\mathrm{j}=1}^{\mathrm{n}} \frac{B_{t}-C_{t}}{(1+\mathrm{R})^{t}}=0
$$

sendo $\mathrm{Bt}-\mathrm{Ct}=$ benefícios econômicos do sistema produtivo em avaliação; 


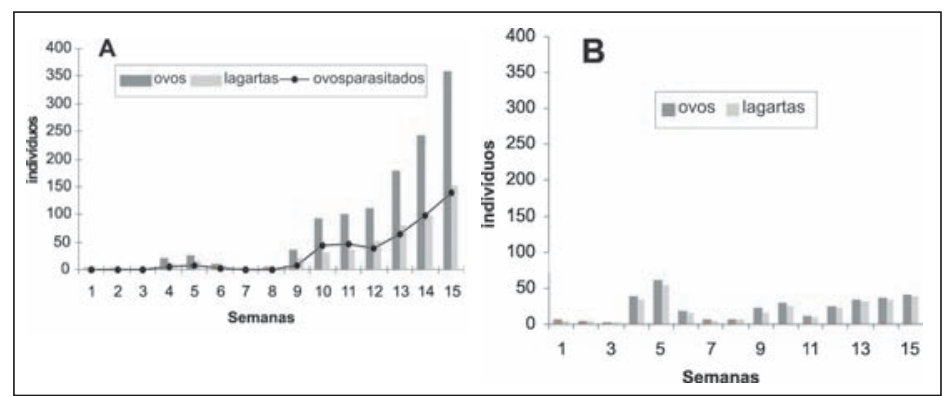

Figura 1. Número de ovos da traça-do-tomateiro coletados em 50 folíolos de tomateiro por semana e o número de lagartas e ovos parasitados por $T$. pretiosum em tomate cultivado em ambiente protegido por controle biológico (A) e por controle químico (B). Luziânia, Embrapa Hortaliças, 1999-2000.

$\mathrm{j}=$ ano inicial do fluxo de benefícios; $\mathrm{n}=$ ano final do fluxo, ou horizonte de liquidação do projeto; $\mathrm{R}=$ taxa interna de retorno.

O valor presente líquido (VPL) ou valor atual líquido foi obtido pela fórmula:

$$
V P L=\sum_{j=1}^{n} \frac{B_{t}}{(1+i)^{t}}-\sum_{j=1}^{n} \frac{C_{t}}{(1+i)^{r}}
$$

sendo $\mathrm{Bt}=$ quantidade produzida multiplicada pelo respectivo preço do produto; $\mathrm{C}_{\mathrm{t}}=$ custo de produção, calculado pela quantidade de fatores utilizados multiplicada pelo respectivo preço do fator; $\mathrm{t}=$ número de anos de investimento, $\mathrm{n}=$ ano final do fluxo de benefícios, $\mathrm{j}=$ ano de início do empreendimento; $i=$ taxa de juros real anual de $6 \%$ (remuneração atual do capital com base nos rendimentos atuais da caderneta de poupança, que é a aplicação mais popular do mercado financeiro). O índice de lucratividade (IL) representa um critério para seleção de alternativas econômicas (Laponni, 1996). Com base neste critério um determinado projeto de produção deverá ser aceito se o IL $>0$, desta forma calcula-se o IL pela seguinte fórmula:

$I L=\frac{\sum_{=1}^{\infty} A^{*}(1+K)^{-1}-I}{I}=\frac{\sum_{I=1}^{\infty} A^{*}(1+K)^{-1}}{I}-1 \therefore I L=\frac{V P L}{I}+1$

em que VPL= Valor Presente Líquido e I= investimentos iniciais.

Os valores dos benefícios e custos foram calculados ano a ano, dentro do horizonte de planejamento do negócio (5 anos). O índice de lucratividade foi determinado. As análises intertemporais foram processadas pelo programa Farmod for Windows desenvolvido e utilizado pelo BIRD (Ace et al., 1995) para avaliação de projetos de desenvolvimento.

\section{RESULTADOS E DISCUSSÃO}

O número de ovos da traça-do-tomateiro foi maior na casa de vegetação que usou o controle biológico (Figura 1), apresentando um crescimento populacional acentuado a partir da $9^{\mathrm{a}}$ semana. $\mathrm{O}$ índice de parasitismo nos ovos da traça-do-tomateiro alcançou 49\%. Haji et al. (1995) encontraram índices de parasitismo da traça-do-tomateiro de até 43\% (em 1990) e 49\% (em 1991) a campo em tomate para processamento industrial, e de até $68 \%$ em casas de vegetação em 1990 no Submédio São Francisco-PE. Villas Bôas e França (1996), em trabalho semelhante, encontraram um índice de parasitismo de $24 \%$ em casa de vegetação. Portanto, o nível de parasitismo observado no presente trabalho foi superior às condições anteriormente observadas na região Centro-Oeste.

A produtividade total estimada em oito colheitas foi de 25.235 frutos com controle químico e 25.420 frutos com controle biológico. Avaliou-se o nível de danos nos frutos causados por pragas em $6 \%$ no controle químico e de $19 \%$ no controle biológico. Os danos observados não afetaram a qualidade do produto a ponto de comprometer o padrão comercial exigido pelo mercado. Desta forma, obteve-se a produção co- mercial de $4.100 \mathrm{~kg}$ com controle químico e de $3.696 \mathrm{~kg}$ com controle biológico. O produto foi comercializado em bandejas de $600 \mathrm{~g}$, na quantidade de 6.833 embalagens para o controle químico e 6.160 para o controle biológico. Em ambos tratamentos, a colheita de frutos foi realizada durante oito semanas (Tabela 1).

Sob o ponto de vista técnico-econômico, ambos os sistemas de controle foram eficientes. Os indicadores obtidos das análises evidenciaram a eficiência do controle biológico. O preço médio recebido pelo produtor foi de $\mathrm{R} \$$ 1,65 por bandeja de $600 \mathrm{~g}$, cobertas por filme PVC. Os custos operacionais de produção no sistema de controle biológico foram de $\mathrm{R} \$ 3,883$, aproximadamente $21,17 \%$ menor do que no sistema sob controle químico. Quando os dois sistemas são comparados, verificase que os gastos do controle biológico foram significativamente menores em inseticidas $(-526,40 \%)$; serviços $(-36,84 \%)$, outros $(-3,4 \%)$. Apesar da produtividade do sistema de controle biológico ter sido menor $(-10,93)$, o custo unitário foi menor, em razão da redução de custos com inseticidas e mão-deobra e outros.

O custo total inicial do controle biológico em que o produtor preferiu instalar seu próprio criatório de $T$. pretiosum foi bastante onerado pelos custos fixos decorrentes de investimentos da estrutura de criação do parasitóide, ainda assim o custo total foi menor $(-13,3 \%)$. A reprodução de parasitóides pelos próprios produtores não é uma atividade necessária, uma vez que existem empresas especializadas neste ramo de negócio que produzem em escala os parasitóides e realizam a distribuição a preços razoáveis. Os investimentos alocados na estrutura produtiva aumentaram, conseqüentemente, as depreciações do capital fixo, resultando em custos totais maiores.

O ponto de equilíbrio da produção foi vantajosamente menor, ocorrendo em um patamar significativamente mais baixo $(-21,17 \%)$. Desta forma, o ponto de equilíbrio do sistema de produção com controle biológico foi de 2.354 bandejas e para o controle químico foi de 2.852 bandejas. Esse ponto indica a 
quantidade mínima que o produtor precisa vender para cobrir os desembolsos necessários para produzir a safra em ambos sistemas. Como o custeio da safra do sistema de controle biológico foi mais baixo, é menor a quantidade que necessariamente tem que ser absorvida pelo mercado para que o produtor remunere os fatores alocados no processo produtivo. Neste ponto, a razão entre a receita e os custos variáveis se torna igual a 1. Qualquer valor superior a 1 indica determinado grau de eficiência econômica. Neste caso, o coeficiente de eficiência econômica foi significativo, indicando que cada unidade monetária investida no sistema produtivo, retornou 2,62 ao produtor (Tabela 1). O tratamento com controle biológico proporcionou uma margem de segurança econômica favorável. Isto é, nas condições tecnológicas em que a cultura foi conduzida, o sistema suporta uma quebra de safra da ordem de $61,79 \%$.

Verifica-se que a viabilidade econômica do sistema de controle biológico foi positiva, como confirmada pela favorável taxa interna de retorno de $24,87 \%$. O índice de lucratividade apoia a decisão do produtor na seleção de um empreendimento. No caso do controle biológico com $T$. pretiosum, o índice de lucratividade de 0,5304 satisfaz as condições I>0, indicando que a tecnologia é uma alternativa economicamente viável, portanto um bom negócio. $\mathrm{O}$ valor atualizado dos investimentos no sistema produtivo foi de $\mathrm{R} \$ 6,4 \mathrm{mil}$. O tempo de retorno dos investimentos ocorrerá por ocasião da $2^{\mathrm{a}}$ safra, indicada pelo 'payback simples-PBS' de 1,94, ou seja, com a comercialização da $1^{a}$ safra completa e aproximadamente $94 \%$ da $2^{\text {a }}$ safra, os investimentos iniciais estarão liquidados. O PBS é uma medida de riscos do empreendimento, quanto maior for o PBS, maior será o risco do negócio. O PBS não é uma medida de rentabilidade dos investimentos, mas é uma medida de liquidez do negócio, ou seja, quanto menor o prazo de recuperação de capital, maior a liquidez do projeto (Laponni, 1997).

A redução da dependência do produtor do mercado de insumos, pela substituição dos sintéticos pela própria criação do parasitóide representa uma gran-

Tabela 1. Indicadores econômicos para produção de tomate com tratamentos de controle químico e controle biológico da traça do tomateiro em casa de vegetação. Luziânia, Embrapa Hortaliças, 1999-2000.

\begin{tabular}{|c|c|c|c|}
\hline \multirow[b]{2}{*}{ Indicadores* } & \multicolumn{2}{|c|}{ Sistemas } & \multirow{2}{*}{$\begin{array}{l}\text { Dife renciais } \\
\text { C. biológicc } \\
(\%)\end{array}$} \\
\hline & $\begin{array}{l}\text { C. biológico } \\
\text { (unidades) }\end{array}$ & $\begin{array}{l}\text { C. químico } \\
\text { (unidades) }\end{array}$ & \\
\hline Sementes $(R \$)$ & 891 & 891 & 0 \\
\hline Fertilizantes $(\mathrm{R} \$)$ & 652,79 & 652,79 & 0 \\
\hline Substrato $(R \$)$ & 234 & 234 & 0 \\
\hline Fungicidas $(R \$)$ & 254,46 & 254,46 & 0 \\
\hline Inseticidas ( $R \$$ ) & 109,50 & 685,91 & $-526,40$ \\
\hline Serviços $(\mathrm{R} \$)$ & 570,00 & 780 & $-36,84$ \\
\hline Outros $(\mathrm{R} \$)$ & $1.171,82$ & $1.207,47$ & $-3,04$ \\
\hline Custos operacionais & $3.883,57$ & $4.705,63$ & $-21,17$ \\
\hline Custo unitário & 0,63 & 0,69 & $-9,45$ \\
\hline Custos fixos & $1.751,77$ & $1.678,40$ & 4,19 \\
\hline Custo total (R\$) & 5.635 & 6384 & $-13,29$ \\
\hline Produtividade ( bandejas de $600 \mathrm{grs}$ ) & 6.160 & 6833 & $-10,93$ \\
\hline Preço recebido pelo produtor / bandeja $(R \$)$ & 1,65 & 1,65 & 0 \\
\hline Receita Bruta $(\mathrm{R} \$)$ & $10.164,00$ & $11.274,45$ & $-10,93$ \\
\hline Eficiência Econômica (UM) & 2,62 & 2,40 & 8,30 \\
\hline Benefício/custo (\%) & 161,72 & 139,59 & 13,68 \\
\hline Ponto de equilíbrio (bandejas de $600 \mathrm{gr}$.) & 2.354 & 2852 & $-21,17$ \\
\hline Margem de segurança (\%) & $-61,79$ & $-58,26$ & 5,71 \\
\hline Taxa intema de retorno (\%) & 24,87 & 39,65 & $-59,42$ \\
\hline Valor Presente Líquido (Mil R\$) & 6,477 & 10,000 & $-54,40$ \\
\hline Pay back (safras) & 1,94 & 1,52 & 21,82 \\
\hline Estrutura de cultivo protegido ( $R \$)$ & $10.011,00$ & $10.011,00$ & 0 \\
\hline Estrutura de criatónio de parasitóides $(R \$)$ & $1.850,00$ & 0 & 100,00 \\
\hline Índice de lucratividade & 1,5304 & 1,9979 & $-31,54$ \\
\hline
\end{tabular}

*Preço pago ao produtor e preços de fatores atualizados em 20 de março de 2006.

de vantagem. Villas Bôas \& França (1996) observaram que a substituição de agrotóxicos pela liberação de $T$. pretiosum, combinada com aplicações semanais de $B$. thuringiensis rendeu a mesma produção, com um custo $20 \%$ menor.

Neste trabalho, os aspectos técnicos e econômicos foram quantificados. Entretanto, os alimentos produzidos com controle biológico concentram atributos positivos que não foram dimensionados. Dentre esses, ressalta-se a segurança alimentar. Neste aspecto deve-se considerar que os alimentos produzidos com controle biológico, sem agrotóxicos, são mais saudáveis para os trabalhadores e consumidores, portanto poderiam ser diferenciados e comercializados com preços mais elevados pela agregação de valor como alimentos saudáveis Os benefícios da produção biológica devem analisados não somente sob o ponto de vista econômico, mas também devem ser re- conhecidos pela sociedade, como alimentos saudáveis, cuja produção contribui para redução de riscos à saúde humana e de impactos ao meio ambiente.

\section{AGRADECIMENTOS}

Os autores agradecem a colaboração do produtor, Sr. Humberto Pante, do auxiliar de laboratório da Embrapa Hortaliças, Sr. José Gomes Teixeira, e a Dra Geni L. Villas Bôas pela revisão do manuscrito e ao Dr. Leonardo B. Giordano pela elaboração do "abstract".

\section{REFERÊNCIAS}

ACE E; GUTTERMAN P; SCHULER C. 1995. Farmod for Windows: software. Washington: BIRD.

ANDREWS PK; REGANOLD JP. 2000. Research networking to evaluate the sustainability of horticultural production systems. Acta Horticulturae 638: 359-368. 
ANTLE J; STOORVOGEL J. 2005, 08 de março. Incorporating systems dynamics and spacial heterogeneity in integrated Assessment of Agricultural Production Systems. Disponível em http:// www.tradeoffs.montana.edu/pdf/ integradassesment.pdf

FRANÇA FH. 1993. Por quanto tempo conseguiremos conviver com a traça-do-tomateiro. Horticultura Brasileira 11: 176-178.

FRANÇA FH; VILLAS BÔAS GL; CASTELO BRANCO M; MEDEIROS MA. 2000. Manejo integrado de pragas. In: SILVA JBC; GIORDANO LB (eds). Tomate para processamento industrial. Brasília: Embrapa Comunicação para Transferência de Tecnologia: Embrapa Hortaliças. p. 112-127.

HAJI FNP; FREIRE LCL; ROA FG; SILVA CN; SOUZA JUNIOR MM; SILVA MIV. 1995. Manejo integrado de Scrobipalpuloides absoluta (Polvony) (Lepidoptera: Gelechiidae) no Submédio São Francisco. In: SOCIEDADE ENTOMOLÓGICA DO BRASIL, 24 Anais.. Jaboticabal: 587-591.
HAJI FNP; VELASQUEZ JJ; BLEICHER E; ALENCAR JA; HAJI AT; DINIZ RS. 1998. Tecnologia de produção massal de Trichogramma spp. Petrolina: EmbrapaCPATSA. 24p.

HIRSCHFELD H. 1998. Engenharia econômica e análise de custos. São Paulo: Atlas. 408p.

LAIARD R; GLAISTER S. 1996. Cost-benefit analysis. New York: Cambridge. 486p.

LAPONNI JC. 1996. Avaliação de projetos de investimentos. São Paulo: Unidas. 266p.

PEARCE DW. 1983. Cost-benefit analysis. London: Macmillan.112p.

PERRIN RK; WIKELMANN DL; MOSCARDI ER; ANDERSON JR. 1985. Formulación de recomendaciones a partir de datos agronômicos: un manual metodológico de evaluación económica. México: Centro Internacional de Mejoramento de Maiz y Trigo. 56p. (Folheto de Información, 27).
SCOLARI DG; COSTA MEFA; SOUZA MC 1985. Programa de análise econômica através de orçamentação parcial (ANECOR). Planaltina: EMBRAPA-CPAC. 43p (EMBRAPA-CPAC. Documentos, 13).

SNODGRASS MM; WALLACE LT. 1993. Agriculture economics and resources management. New Jersey: Prentice Hall. 521p.

VILLAS BÔAS GL; FRANÇA FH. 1996. Utilização do parasitóide Trichogramma pretiosum no controle da traça-do-tomateiro em cultivo protegido de tomate. Horticultura Brasileira 14: $223-225$. 